\title{
Scaling with microRNAs
}

Once dendrites have established coverage of their receptive fields during development, growth of the dendritic arbour in synchrony with body growth (dendritic scaling) is essential to maintain the coverage. How this proportional growth of dendrites is coordinated is largely unknown. A study by Jan and colleagues now shows that expression of the microRNA gene bantam in epithelial cells regulates dendritic scaling in peripheral Drosophila melanogaster class IV dendritic arbour (da) neurons through downregulation of Akt signalling.

Focusing on class IV da neurons, because of their elaborate arbours, the authors screened D. melanogaster mutants for defects in dendritic growth, and identified the most severe effects in flies lacking bantam. Class IV da neurons in these mutants exhibited disproportional dendritic growth during scaling. Not only were the dendrites longer than normal in these cells, they were greater in number and density as well.

The authors then monitored dendrite growth throughout larval development. The da neurons of bantam mutants showed normal dendritic growth during the early, rapid growth phase, when receptive fields are established; however, shortly afterwards their dendritic arbours grew larger than those of wild-type da neurons. This suggests that bantam regulates signals that normally restrict dendrite growth.

bantam expression was detectable in wild-type larval muscle and epithelial cells as well as in peripheral neurons after receptive fields had been established. Surprisingly, knocking down bantam in single class IV da neurons had no effect on scaling. Moreover, neuronal expression of bantam in the bantamnull flies did not rescue the scaling defect. However, when bantam was expressed in bantam-null epithelial cells the scaling defect was reduced, and the extent of the rescue was dependent on the level of bantam expression. In addition, overexpression of bantam in wild-type epithelial cells severely reduced dendrite growth. These results are consistent with bantam in epithelial cells regulating dendrite growth-inhibitory signals.

Which growth signals are regulated by bantam? Using expression profiling, the authors identified Akt - a well-established regulator of growth - among the numerous candidate genes that were deregulated in neurons of bantamdeficient flies, as Akt expression was increased in these neurons but was normal when bantam was expressed in epithelial cells. Consistent with these results, overexpression or activation of Akt in neurons caused scaling defects similar to those exhibited by bantam mutants. Conversely, knockdown or inhibition of Akt reduced dendritic growth. This indicates that bantam regulates Akt levels in a non-cellautonomous manner in da neurons.

The authors also showed that bantam regulates the growth of Class III, but not Class I, da neurons. They suggest that the different time courses of dendritic scaling of these cells might mean that they require different scaling signals. It remains to be seen whether noncell-autonomous regulation through microRNAs is a general mechanism for coordinated dendritic scaling.

Claudia Wiedemann

ORIGINAL RESEARCH PAPER Parrish, J. Z., $\mathrm{Xu}$, P. et al. The microRNA bantam functions in epithelial cells to regulate scaling growth of dendrite arbors in Drosophila sensory neurons. Neuron 24 Sep 2009 (doi:10.1016/j. neuon.2009.08.006) FURTHER READING Kosik, K. S. MicroRNAs tell an evo-devo story. Nature Rev. Neurosci. 10, 754-759 (2009)
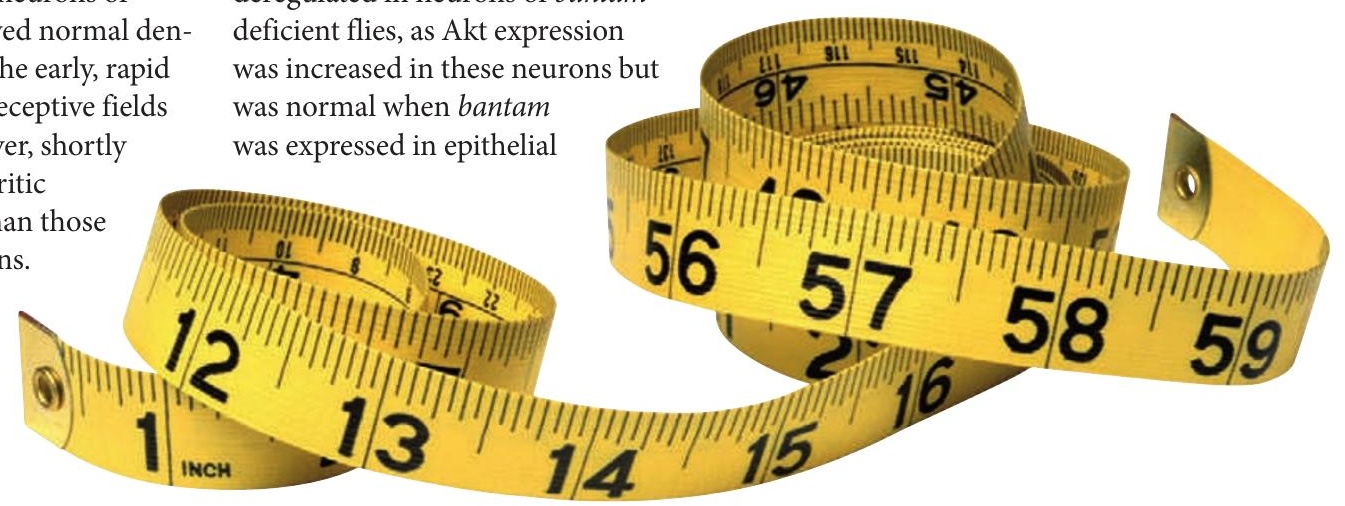